\title{
RETRIEVAL OF LOCAL CLIMATE ZONES AND THEIR LINKAGES TO LAND SURFACE TEMPERATURE USING REMOTE SENSING IN HARARE METROPOLITAN CITY, ZIMBABWE
}

\author{
Terence Darlington Mushore ${ }^{1,2, *}$ \\ ${ }^{1}$ Physics Department, University of Zimbabwe, P.O. Box MP167, Mount Pleasant, Harare, Zimbabwe \\ (tdmushore@mail.com) \\ ${ }^{2}$ Discipline of Geography, School of Agricultural, Earth and Environmental Sciences, University of KwaZulu-Natal, P/Bag \\ X01, Scottsville, Pietermaritzburg 3209, South Africa
}

KEYWORDS: Data Scarcity, Satellite Data; Temperature Variations; Climate; Urban Land Use; Spatial Tools; Urban Heat Island

\begin{abstract}
This study sought to determine Local Climate Zones (LCZs) in Harare metropolitan City, using Landsat 8 multi-spectral and multi-temporal data. The World Urban Database and Access Portal Tool (WUDAPT) and Support Vector Machine classifiers were applied. Training datasets were extracted from Google Earth as prescribed by the WUDAPT procedure. Before image classification, we tested the separability of the LCZs, using the Transformed Divergence Separability Index (TDSI) based on the digitized training datasets and Landsat 8 data. Derived LCZs were then linked with Landsat 8 derived Land Surface Temperature (LST) for the cool and hot seasons. TDSI values greater 1.9 were obtained indicating that LCZs were highly separable. Comparatively, the WUDAPT method produced more accurate LCZs results (Overall accuracy $=95.69 \%$ ) than the SVM classifier (Overall accuracy $=89.86 \%$ ) based on seasonal Landsat 8 data. However, SVM derived accuracies were within the acceptable range of at least $80 \%$ (overall accuracy) in literature. Further, LST was observed to be high in LCZs with high built-up density and low vegetation proportion, when compared to other zones. Due to high proportion of vegetation, sparsely built areas were at least $1^{\circ} \mathrm{C}$ cooler. Although LCZs are usually linked at $2 \mathrm{~m}$ air temperature, they also strongly explain LST distribution. This work provides insight into the importance of mapping LCZs in third world countries where such information remains scarce.
\end{abstract}

\section{INTRODUCTION}

There is a global call for studies which use LCZs in urban areas for input into World Urban Database and Access Portal Tools (WUDAPT) (Ching et al., 2018). Currently, progress in urban climate science is retarded by lack of useful information that describes aspects of the form and function of cities at detailed spatial resolution (Bechtel, et al., 2015). WUDAPT is being developed to gather and disseminate LCZs information. Bechtel, et al. (2015) developed WUDAPT protocols, which are easy to understand, use freely available data such as medium resolution remote sensing imageries`and can be applied by someone with limited knowledge in spatial analysis or urban climate science. There is a severe lack of studies that map LCZs in urban areas of Africa and more specifically in Zimbabwe. To date only the LCZ map for Khartoum, Sudan has been completed and successfully shared on the WUDAPT website (http://www.wudapt.org/cities/in-africa/).

In view of the above, the objectives of this study are i) to contribute to WUDAPT by using Landsat data to map LCZs of Harare metropolitan city in Zimbabwe, ii) to compare the WUDAPT procedure (which uses Random Forest classifier) with the Support Vector Machines (SVM) classifier for improving LCZ mapping accuracy and iii) to link the spatial structure of LCZs with land surface temperature in different seasons in Harare.

\subsection{Description of study area}

This study was conducted in Harare, the capital and largest city of Zimbabwe in Southern Africa (Figure 1). The climate is generally humid with an average temperature of $18^{\circ} \mathrm{C}$ and mean annual rainfall of $850 \mathrm{~mm}$ (Torrance, 1981). The hot season (mid-September to mid-November) was chosen in this study because it is the warmest period hence ideal for understanding extreme thermal conditions (Manatsa et al., 2013).

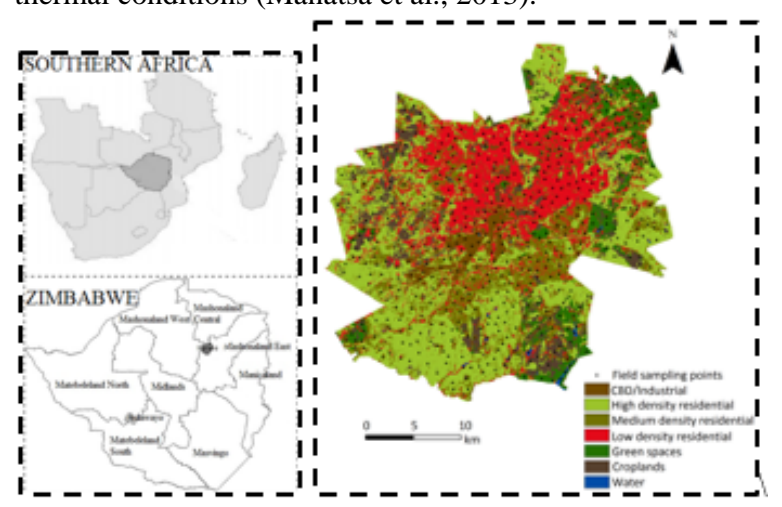

Figure 1: Location of the area under study area and field sample collection points

Table 1 provides description of major land use and land 
cover classes in Harare (Mushore et al., 2017). High density built-up areas dominate the south-western half of the city, which include the Central Business District, industrial areas and high density settlements. Low and medium density built-up areas mostly occupy the northeastern residential suburbs (Wania, et al., 2014; Mushore et al., 2016). According to Wania, et al. (2014), 'leafy' suburbs in the north are mainly occupied by high income strata while low income strata populate the high density residential areas in the south.

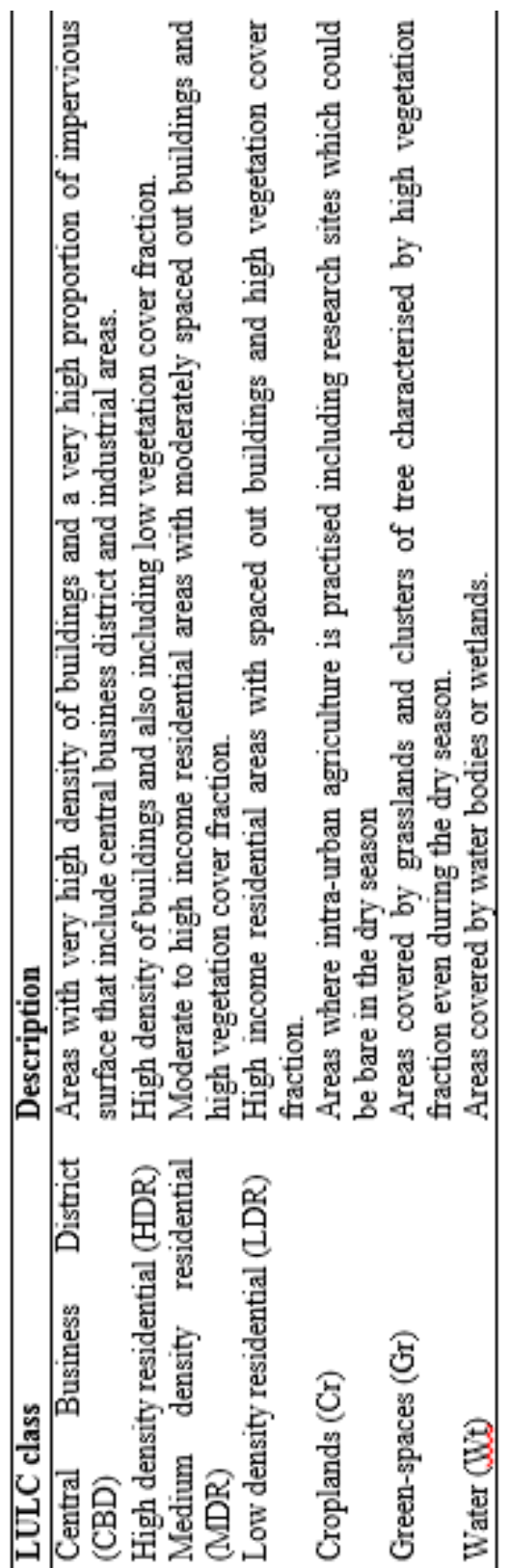

Table 1: Description of general land use and land cover types identified in Harare

\section{METHODOLOGY}

2.1 Remote sensing data collection and pre-
processing
Cloud-free Landsat 8 imagery of path/row $170 / 72$

acquired in 2017 during the cool and hot seasons on dates shown in Table 2 were used. The rainy and post rainy seasons were avoided due to unavailability of cloud free images. The imagery were downloaded free of charge from the United States Geological Survey's (USGS) earth explorer website. Three dates per season were used to incorporate intra- and inter-seasonal Local Climate Zone variations into mapping procedure.

\begin{tabular}{|c|c|c|c|c|}
\hline \multirow[t]{2}{*}{ Date acquired } & \multirow[t]{2}{*}{ Season } & \multicolumn{3}{|c|}{ Average weather conditions during acquisition hour } \\
\hline & & $\begin{array}{c}\text { Air temperature } \\
\left({ }^{\circ} \mathrm{C}\right)\end{array}$ & $\begin{array}{c}\text { Relative Humidity } \\
(\%)\end{array}$ & $\begin{array}{c}\text { Wind speed } \\
(\mathrm{m} / \mathrm{s})\end{array}$ \\
\hline 17 June 2017 & Cool & 17.0 & 51.7 & 2.8 \\
\hline 19 July 2017 & Cool & 17.7 & 45.0 & 1.8 \\
\hline 20 August 2017 & Cool & 16.8 & 49.3 & 2.9 \\
\hline 21 September 2017 & Hot & 22.3 & 42.8 & 2.9 \\
\hline 23 October 2017 & Hot & 26.0 & 26.8 & 3.2 \\
\hline 8 November 2017 & Hot & 29.2 & 15.7 & 2.5 \\
\hline
\end{tabular}

Table 2: Cloud-free Landsat 8 imageries used for LCZ and LST retrieval in this study

\subsection{Digitizing of classification training samples and} LCZ separability test

For each LCZ class, representative samples were digitized from Google Earth in the form of polygons in line with the WUDAPT methodology, guided by knowledge of the study area and field survey conducted. The sample representing each class was digitized from fairly distributed locations across the study area in order to incorporate intra-class variability. Evaluation of the suitability of the training samples digitized from Google Earth to perform classification was done by statistically testing for separability using the transformed divergence separability index. The Transformed Divergence Separability Index (TDSI) was used to test the extent to which LCZs can be distinguished from each other using Landsat 8 multispectral data. TDSI ranges between 0 and 2; the closer to 2, the better the classes can be separated from each other. Values of TDSI below 1 imply that the two classes being compared are not easy to separate, using a given remote sensing dataset. In such cases classes whose TDSI is low may be combined to allow further tests until appreciable values are obtained and classes can be easily distinguished using remote sensing. LCZ mapping would only proceed if the zone types in the study area were acceptably separable using the digitized training LCZ samples and Landsat 8 datasets.

\subsection{Retrieval of local climate zones from Landsat data}

Classification of local climate zones is split into two sub procedures namely (i) Digitizing of training sites and preclassification test for class separability and ii) Local climate zone classification by applying two classifiers (Random Forest - RF and Support Vector machines SVM) and statistically comparing the accuracy in mapping. The separability test was done to ensure that the classes are clearly distinct. High separability between classes was meant to provide confidence to proceed with the classification. Land surface temperature retrieval A single channel approach using Band 10 of Landsat 8 was used to retrieve land surface temperature (LST) from thermal infrared data for each of the 6 imagery scenes. 
The raw thermal infrared digital numbers were converted to LST by adopting steps used by Weng et al. (2004). The procedure involved conversion of digital numbers to radiances, radiances to brightness temperatures and finally brightness temperature to land surface temperature (emissivity correction). Emissivity maps for the area were obtained as detailed by Sobrino et al. (2004) using normalized difference vegetation index (NDVI) based approach separately for each of the 6 imagery scenes. Similar Sobrino, et al. (2004), NDVI value of 0.2 was used for a purely soil pixel and 0.5 for a purely vegetation pixel. Average land surface temperature maps for the cool and the hot season were computed since three temperature maps were computed per season. In order to establish the link between LCZ and LST, spatial overlay of the two maps and data extraction using the zonal statistics tool in ArcMap10 were used. Using the zonal statistics tool, we retrieved the average LST for each LCZ.

\section{RESULTS AND DISCUSSION}

\subsection{Test of separability of Local Climate Zones}

The transformed divergence separability index ranged between 1.958 and 2. Although "Compact mid-rise" and "Compact low-rise" were the least separable, the TDSI value of 1.958 was very high indicating that the two were highly separable using the training samples for each LCZ type digitized from Google Earth and Landsat 8 data. Overall, all the LCZ were highly separable from each other using the digitized samples as shown by high TDSI values (very close to 2). Due to similarities, Chen et al., (2017) were forced to combine "Heavy industry" with "Large low-rise" LCZ in Taipei because they were not clearly distinct.

\subsection{The LCZ map of Harare}

Figure 2 shows the high accurate LCZ maps of Harare produced by the Support Vector Machines classifier (Figure 2a) and the WUDAPT approach (Figure 2b). Visual inspection show similarities between the maps from both approach especially the general distribution of classes. "Sparsely built" LCZ covered most of the northern half of the city. The "Compact mid-rise" and the "Compact low-rise” LCZ occupied the central part of the city. The southern half is occupied mostly by the "Bush/scrubs" and the "Light-weight low-rise". The "Dense trees" LCZ occupied more area in the northern half than the south. The results concurred with Kamusoko, et al. (2013) who observed that built-up densities are high in the southern half of the city.

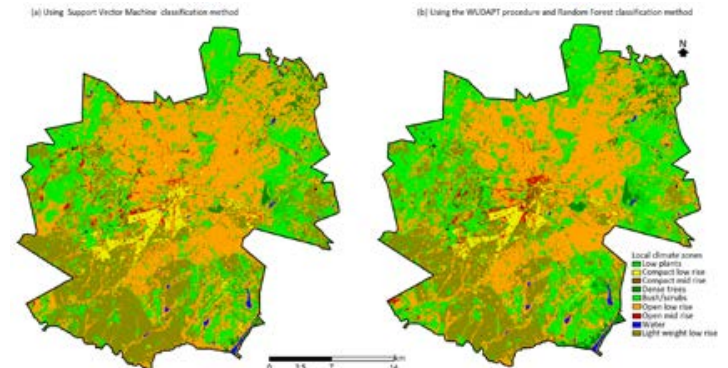

Figure 2: Local climate zones map for Harare metropolitan city obtained using (a) SVM classifier and (b) WUDAPT method (Random Forest classifier)

\subsection{Accuracy of classification per analysis type}

Overall accuracies were greater than $85 \%$ for both methods which indicates very high mapping accuracy. Although both methods mapped LCZ with very high accuracy, the WUDAPT method was more accurate $(\mathrm{OA}=95.7 \%)$ than the SVM $(\mathrm{OA}=89.9 \%)$. The Random Forest based WUDAPT approach was found to be superior to SVM when multi-temporal Landsat 8 from different seasons of the same year were used as input data. The McNemar's Statistic (Z) was 26.02 (>1.96) implying that the difference between WUDAPT method based- and SVM based LCZ mapping was statistically significant at $95 \%$ confidence interval.

\subsection{Average temperature for $L C Z$}

Visual inspection of Figure 3 shows that the northern half was cooler than the southern half in both the cool and hot seasons. For example, in the hot season land surface temperature ranged between 35 and $450 \mathrm{C}$ in the southern areas compared to a range between 20 and $35^{\circ} \mathrm{C}$ in the north-western half of the city. Combining Figure 4 and Figure 3 indicated that very warm areas corresponded with "Compact mid-rise", "Compact low-rise" and "light-weight low-rise" which occupied most of the southern areas. High temperatures were recorded in the “Compact mid-rise” LCZ due to high heat absorption by the concentrated buildings. Walls of tall buildings absorb a lot of heat while closely packed buildings impede heat removal by wind resulting in elevated surface temperatures. High rise buildings also reduce the sky view factor implying reduction in the amount of radiation lost to outer space (Blake et al., 2011) resulting in more heat trapped within built-up area and enhanced surface warming. "Compact low-rise" buildings absorb a lot of heat due to low vegetation fraction and packed buildings. Coolest areas (LST<35oC) coincided with the "Water", "Sparsely built" and "Dense trees" LCZs. The "Water" areas had low daytime surface temperature during the day in both seasons due to high latent heat transfer and evaporation cooling. Sparse built and dense green LCZs had low surface temperature because of abundance of vegetation which according to Odindi, et al. (2015) increase cooling by evaporation. The findings are also in tandem with Perera \& Emmanuel, (2018) who, in Colombo, observed temperature decrease from densely 
built LCZ such as "Cmpact mid-rise" to LCZ type with high density of vegetation such as the "Dense trees" as well as "Water" areas. Chen et al., (2017) cordially also observed that higher urban development and building density are associated higher thermal loads in Taipei.

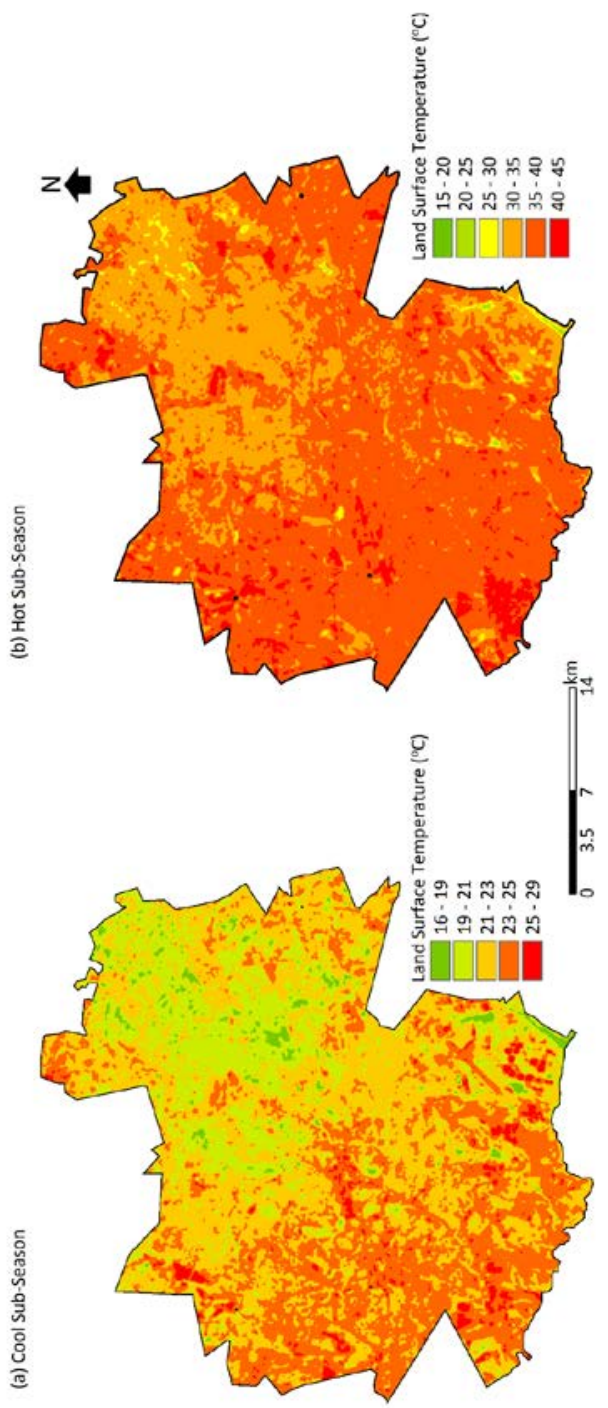

Figure 3: Land surface temperature at coincident time with LCZ map (a) in the cool and (b) the hot season

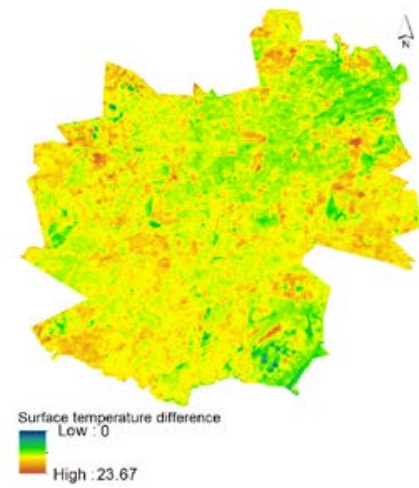

Figure 4: Summer-winter surface temperature difference
Figure 4 shows that the summer-winter temperature difference was higher in built LCZs than in other areas.

As expected, the "Water" and the "Dense trees" LCZs were the coolest with average land surface temperatures of 10 and $19.8^{\circ} \mathrm{C}$, respectively, in the cool season (Figure 5). The "Compact mid-rise" was the warmest $\left(38.8^{\circ} \mathrm{C}\right.$ in the hot and $24.9^{\circ} \mathrm{C}$ in the cool season) followed by the "Compact low-rise" $\left(37.8^{\circ} \mathrm{C}\right.$ in the hot and $23.5^{\circ} \mathrm{C}$ in the cool season) and the "Light weight low-rise" $\left(37.5^{\circ} \mathrm{C}\right.$ in the hot and $23.3^{\circ} \mathrm{C}$ in the cool season). Similarly, in Kochi India, maximum heat intensity was recorded in "Compact mid-rise" while intense cooling was recorded in "Sparsely built" areas in all seasons (Thomas, et al., 2014).

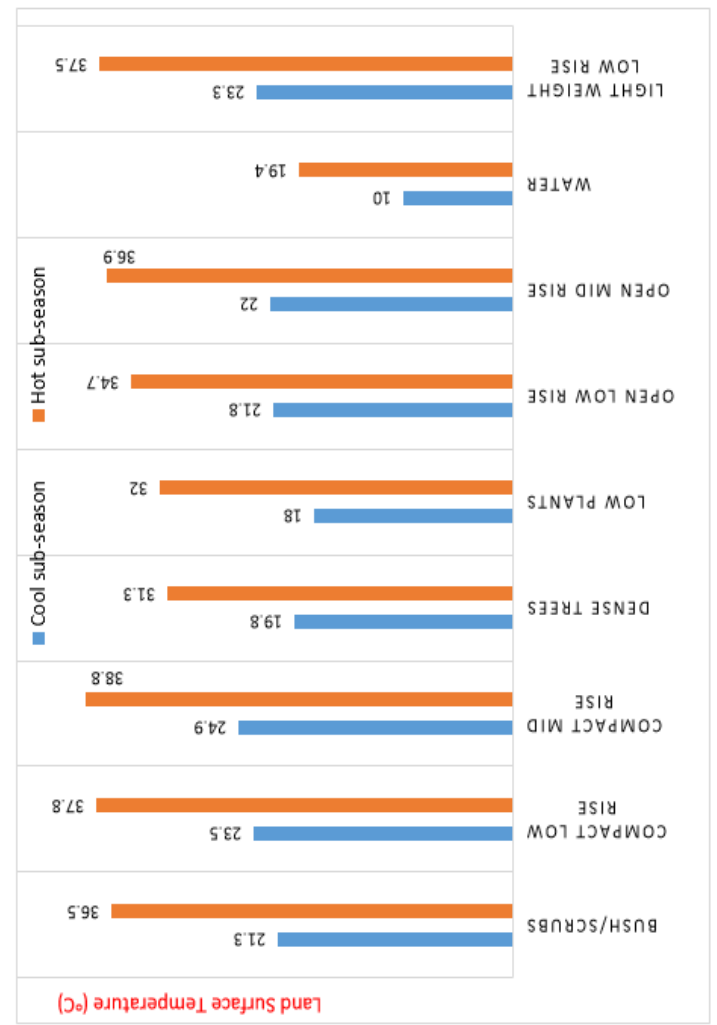

Figure 5: Relationship between LCZ and LST in Harare for the cool and hot seasons

\section{CONCLUSIONS AND RECOMMENDATIONS}

WUDAPT procedure in comparison to the SVM classifier using Landsat 8 multi-spectral and multitemporal datasets were applied to determine and map the Harare Metropolitan City's LCZs. Nine LCZ types were identified and analysis showed that the classes were highly separable. Results showed that both the WUDAPT procedure and the SVM based approach mapped LCZ for Harare with acceptable high levels of accuracy. Intense heating was recorded in high impervious and densely built-up LCZs while intense cooling was observed in sparsely built, densely vegetated and water bodies’ LCZs. The study recommends that more research should be done to continuously improve the accuracy of the 
procedure by testing potential of new datasets and classifiers.

\section{LIST OF REFERENCES}

Chen, Y., Lin, T. P., \& Lin, Y. C. T. (2017). Modeling the urban thermal environment distributions in Taipei Basin using Local climate zone ( LCZ ) ( LCZ ), (March), $31-34$.

Mushore, T. D., Odindi, J., Dube, T., \& Mutanga, O. (2017). Understanding the relationship between urban outdoor temperatures and indoor air-conditioning energy demand in Zimbabwe. Sustainable Cities and Society, 34(June),

https://doi.org/10.1016/j.scs.2017.06.007

Bechtel, B., Alexander, P. J., Böhner, J., Ching, J., Conrad, O., Feddema, J., Mills, G., See, L., \& Stewart, I. (2015). Mapping local climate zones for a worldwide database of the form and function of cities. ISPRS International Journal of Geo-Information, 4(1), 199-219.

Ching, J., Mills, G., Bechtel, B., See, L., Feddema, J., Wang, X., Ren, C., Brousse, O., Martilli, A., Neophytou, M. and Mouzourides, P., (2018). World Urban Database and Access Portal Tools (WUDAPT), an urban weather, climate and environmental modeling infrastructure for the Anthropocene. Bulletin of the American Meteorological Society, (2018).

Kamusoko, C., Gamba, J., \& Murakami, H. (2013). Monitoring Urban Spatial Growth in Harare Metropolitan Province, Zimbabwe. Advances in Remote Sensing, 2 322-331.

Manatsa, D., Morioka, Y., Behera, S. K., Yamagata, T., \& Matarira, C. H. (2013). Link between Antarctic ozone depletion and summer warming over Southern Africa. Nature Geoscience, 6, 934-939.

Thomas, G., Sherin, A., Ansar, S., \& Zachariah, E. (2014). Analysis of urban heat island in Kochi, India, using a modified local climate zone classification. Procedia Environmental Sciences, 21, 3-13.

Torrance, J. (1981). Climate handbook of Zimbabwe. Zimbabwe Meteorological Services, Harare.

Wania, A., Kemper, T., Tiede, D., \& Peter Zeil, b. (2014). Mapping recent built-up area changes in the city of Harare with high resolution satellite imagery. Applied Geography, 46, 35-44.

Blake, R., York, N., Curitiba, A. G., Tokyo, T. I., Horton, R., \& York, N. (2011). Urban climate: Processes, trends and projections. First Assessment Report of the Urban Climate Change Research Network, 43-81.

Perera, N. G. ., \& Emmanuel, R. (2018). A “Local
Climate Zone” based approach to urban planning in Colombo, Sri Lanka. Urban Climate, 23, 188-203. https://doi.org/10.1016/j.uclim.2016.11.006 